\title{
Relpio: uma aplicação Mobile para auxiliar no cadastro de vítimas de catástrofes naturais
}

\author{
Relpio: a Mobile application to support victims in natural disaster \\ Anrafel Fernandes Pereira*+, Diogo Padilha†, Leonardo Lima Procópio*
}

\begin{abstract}
Resumo
A preparação para catástrofes não é mais uma escolha, mas sim um caminho obrigatório, independentemente de onde se vive. Sendo assim, desenvolver e buscar apoio em recursos tecnológicos é de extrema importância para ajudar a minimizar os impactos causados por estes eventos. Este artigo apresenta um aplicativo móvel, ainda em fase inicial, que tem como objetivo apoiar o cadastro de vítimas de catástrofes naturais e ainda, apresentar os principais meios para que a população possa ajudar estas famílias. Um cenário real de catástrofe natural ocorrido nas cidades de Paraíba do Sul e Três Rios, ambas localizadas no interior do estado do Rio de Janeiro apoiou o desenvolvimento desta solução. A aplicação desenvolvida é chamada de Relpio.
\end{abstract}

Padilha D, Procópio LL. Relpio: uma aplicação Mobile para auxiliar no cadastro de vítimas de catástrofes naturais. Revista Eletrônica Teccen. 2016 Jan./Jun.; 09 (1): 20-25.

Palavras-Chave: Mobile; Emergência; Catástrofes Naturais; Comunidade Inteligente.

\begin{abstract}
Disaster preparedness is no longer a choice but a mandatory precaution, no matter where people live. Thus, development and support in technological resources is of the utmost importance to help minimize the impacts of these events. This article presents a mobile application, still in the initial phase, which aims to support the registration of natural disaster victims and also present the main means available for the population to help the affected families. A real scenario of natural disaster occurred in the cities of Paraiba do Sul and Três Rios, both located in the state of Rio de Janeiro has supported the development of this solution. The developed application is called Relpio.

Keywords: Mobile; Emergency; Natural disasters; Intelligent Community.
\end{abstract}

\section{Introdução}

Nos últimos tempos, tem-se percebido com frequência que não existe uma cidade, estado ou país que possa resistir a ameaça de uma catástrofe natural, mesmo sendo ameaçados em diferentes níveis. Com isso, cada vez mais os governos e pesquisadores tem buscado mecanismos para minimizar as perdas e os danos oriundos destes tipos de eventos, conforme pode ser visto nos trabalhos de Wattegama (2007), Lage et al. (2011), Cordeiro et al. (2011) e De Faria Cordeiro et al. (2014). Diante deste cenário, é possível perceber ainda que a preparação para catástrofes não é mais uma escolha, mas sim um caminho obrigatório, independentemente de onde se vive.

Em relação aos recursos tecnológicos, cada vez mais as potencialidades das redes e dos dispositivos móveis vem sendo exploradas no sentido de fornecerem um vasto conjunto de serviços e aplicações dos mais diversos tipos. Entre elas, pode-se citar as aplicações que oferecem apoio aos usuários em situações de emergência, conforme pode ser percebido nos trabalhos de Souza \& Kushchu (2005), Education (2010) e Allen, Graupera \& Lundrigan (2010).

Este artigo está inserido em um contexto específico de uma situação de catástrofe ocorrida na região Centro Sul Fluminense do estado do Rio de Janeiro, no final do mês de Janeiro de 2016. Neste evento, devido às fortes chuvas na região, principalmente nas cidades de Paraíba do Sul e Três Rios, ambas situadas às margens do rio Paraíba do Sul, foram afetadas pelo grande volume de água do rio que corta o centro das cidades e por córregos localizados nos bairros, provocando enchentes e inundações em diversos pontos das cidades, deixando várias famílias desabrigadas e as cidades em estado de alerta.

Diante destes tipos de eventos, percebe-se que a ajuda inicial às vítimas, quase nunca chega num tempo inferior a 48 horas (FEMA, 2010). Sendo assim, é

\footnotetext{
Afiliação dos autores: $\uparrow$ Universidade Severino Sombra, Vassouras-RJ, Brasil.

¥ Centro Vocacional Tecnológico Três Rios, FAETEC, Três Rios-RJ, Brasil.

* Endereço para correspondência: Universidade Severino Sombra, Av. Exped. Oswaldo de Almeida Ramos, 280 - Centro - Vassouras, RJ - CEP $27700-000$.

E-mail: anrafel@live.com
} 
fundamental criar mecanismos de auto-organização e autoproteção entre as famílias afetadas, principalmente para auxiliá-las nas primeiras horas após uma catástrofe.

Para isso, neste artigo é apresentada uma aplicação mobile desenvolvida com o objetivo de apoiar as famílias vítimas do evento relatado acima, oferecendo ainda um mecanismo para que órgãos responsáveis tais como defesa civil e assistências sociais, possam oferecer o auxílio necessário para estas vítimas. A aplicação desenvolvida e apresentada neste trabalho é conhecida como Relpio e pode ser acessada através do endereço:https://play.google.com/store/apps/ details?id=br.gdgtresrios.relpio\&hl=pt_BR.

A hipótese levantada neste artigo é a seguinte: $\mathrm{Se}$ oferecermos às famílias vítimas de catástrofes naturais uma aplicação móvel para apoiar a realização do cadastro das famílias afetadas, de forma a coletar informações essenciais da situação destas pessoas logo no primeiro momento, é possível minimizar o tempo de ajuda inicial às vítimas e ainda, direcionar o tipo de ajuda adequada para cada situação e apoio a estas pessoas.

Este artigo está dividido da seguinte maneira: na seção 2, o aplicativo Relpio é apresentado, destacando as suas principais funcionalidades e aspectos de implementação da aplicação. Na seção 3, uma breve avaliação do aplicativo é apresentada, bem como os resultados iniciais e uma discussão sobre o tópico.
$\mathrm{Na}$ seção 3 são apresentadas ainda, as principais contribuições da aplicação, destacando ainda alguns dos possíveis trabalhos futuros. Por fim, na seção 4 são apresentadas as considerações finais deste trabalho.

\section{Aplicativo Relpio}

Para ilustrar o cenário no qual o Relpio pode ser utilizado, a Figura 1 é apresentada. Através dela é possível perceber o contexto no qual a aplicação está inserida. Conforme foi comentado, o objetivo principal da aplicação é servir como um mecanismo para auxiliar que famílias, vítimas da catástrofe natural ocorrida nas cidades de Paraíba do Sul e Três Rios no mês de Janeiro de 2016, possam realizar o seu cadastro solicitando ajuda aos órgãos responsáveis, e ainda, apresentar os principais pontos de apoio para recebimento de doações, de forma que pessoas interessadas em ajudar as vítimas desta catástrofe possam encontrar os locais apropriados para realizarem sua doação.

Ainda sobre a Figura 1, um usuário, identificado como vítima A precisa realizar o seu registro na aplicação. Com a aplicação já instalada no dispositivo móvel, a vítima A clica sobre a opção Cadastro de Vítima (Figura 2) na tela principal da aplicação e a tela de cadastro é aberta para que sejam preenchidas as informações necessárias (Figura 3).

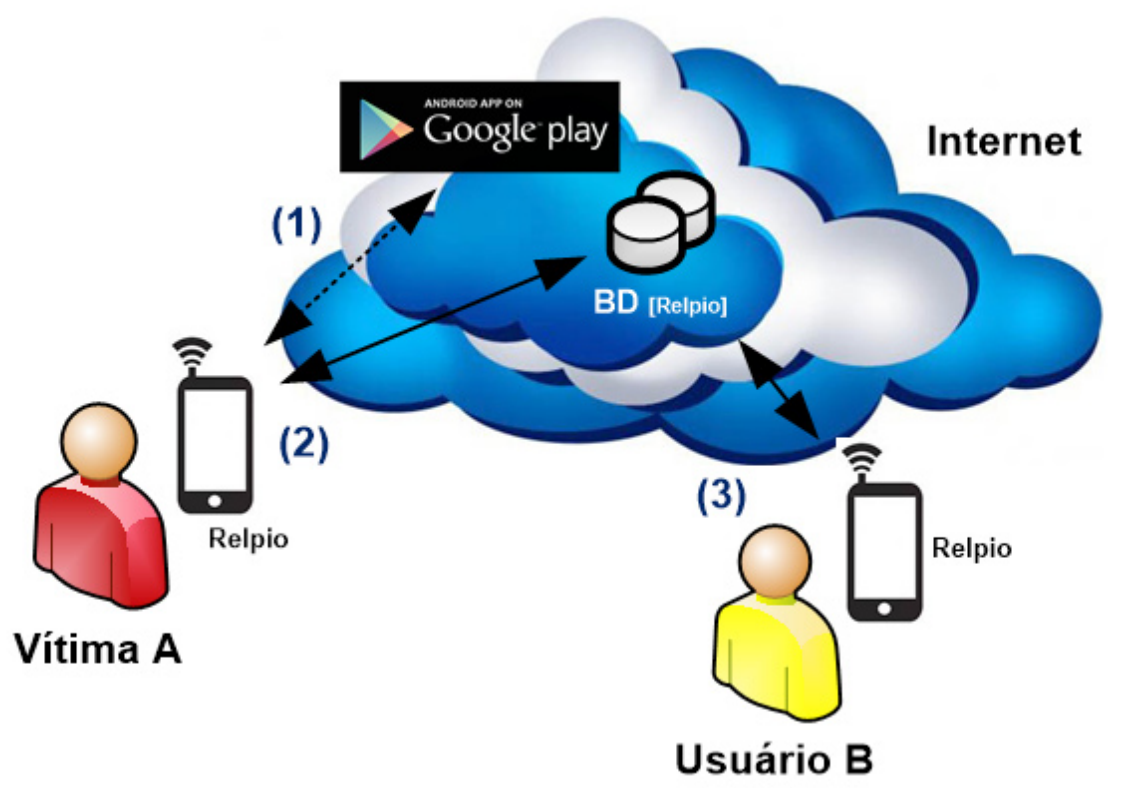

Figura 1. Visão Geral da Aplicação Relpio Fonte: o autor (2016) 


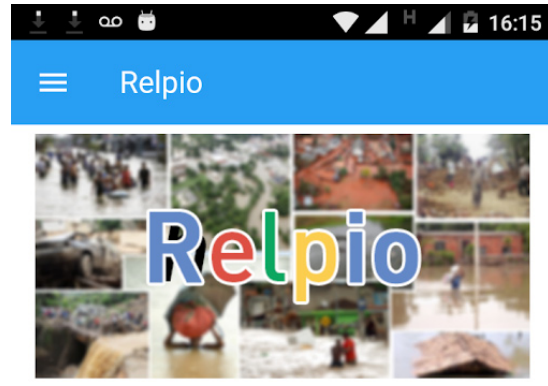

Utilize este aplicativo para solicitar ajuda ou acha os pontos de coleta de material para apoio às vítimas!

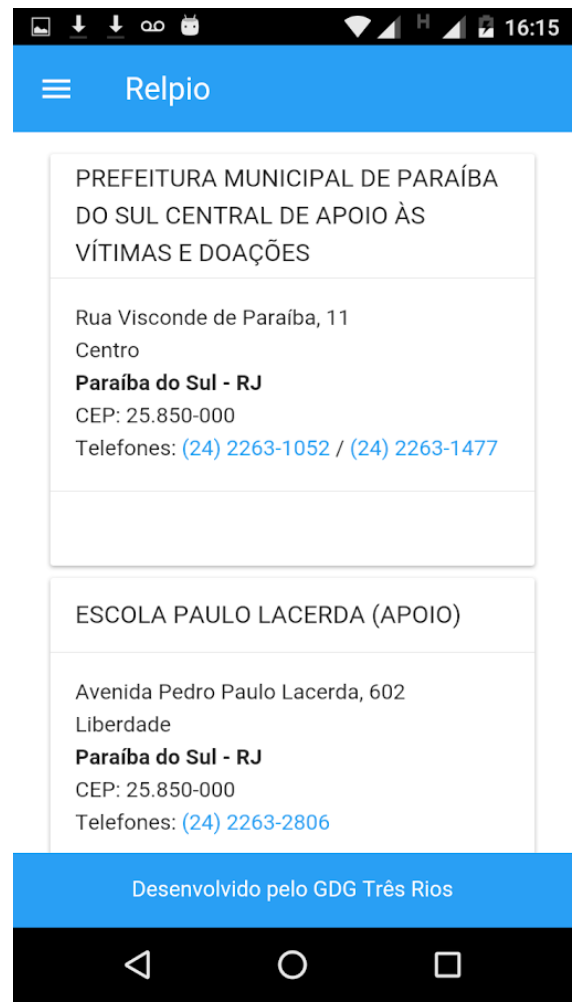

Figura 4. Tela de Pontos de Apoio
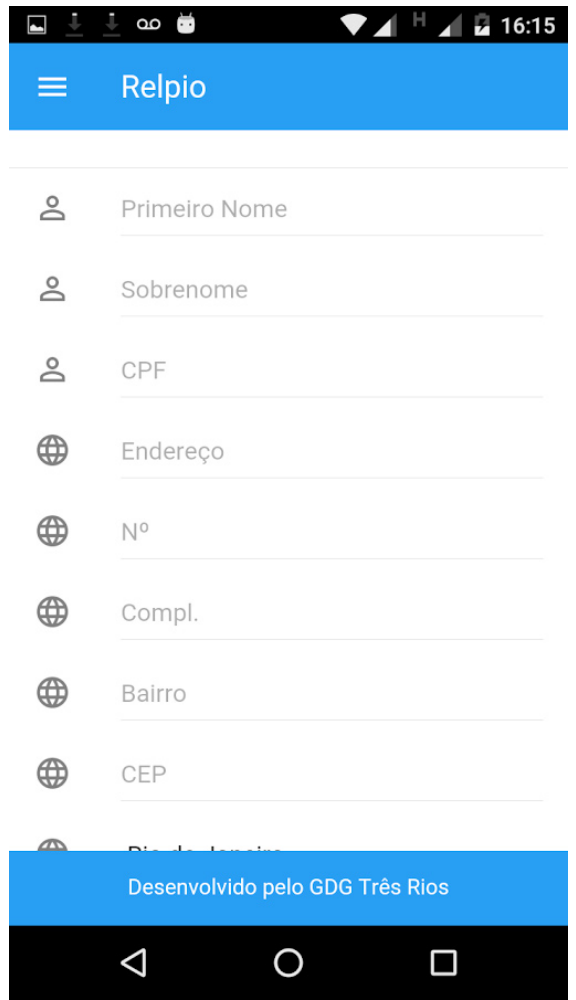

Figura 3. Tela de Cadastro de Vítimas
Algumas destas informações são: nome, CPF, endereço completo, número de residentes na casa, número de pessoas com necessidades especiais, entre outros. Um campo de observação também é disponibilizado neste momento para que a vítima possa preencher com outra informação que ache relevante para aquele momento. Após finalizar o cadastro, a vítima A clica na opção salvar e os dados são gravados na aplicação.

Atualmente, todos os cadastros realizados são armazenados em um banco de dados na nuvem, sendo assim, a conexão com a internet é fundamental para utilização do aplicativo (Figura 1). Depois de cadastradas, as informações são disponibilizadas apenas para os órgãos competentes e responsáveis para auxiliar às vítimas. Ao acessar o botão "Sobre" algumas informações importantes são apresentadas para que órgãos responsáveis possam conhecer a maneira de solicitar acesso a estas informações.

Ainda sobre a Figura 1, outro usuário da aplicação, identificado como Usuário B, deseja realizar uma doação para as vítimas da catástrofe que aconteceu. Para isso, acessando a aplicação o usuário B pode conhecer através da opção Pontos de Apoio, os principais locais em sua cidade para fazer a sua ajuda (Figura 4).

É importante ressaltar que atualmente o cadastro dos pontos de apoio são realizados pelos próprios desenvolvedores, de maneira que possa se manter um controle sobre estes pontos. Entretanto, trabalhos futuros apontam em uma nova direção, sendo discutido nas próximas seções.

A realização do cadastro de vítimas pode ser 
feita pela própria pessoa, vítima do evento. Entretanto, analisando como a abordagem às famílias atingidas foi realizada durante $o$ atendimento às vítimas, percebeuse outro potencial uso da ferramenta. Os próprios órgãos responsáveis, tais como, assistentes sociais, poderiam fazer uso da ferramenta, ficando eles próprios responsáveis pela realização do cadastro das vítimas no momento de abordagem à estas famílias.

Outras observações sobre como a aplicação pode ser utilizada, são destacadas na seção 3 . A seção seguinte apresenta alguns aspectos técnicos de implementação envolvidos na aplicação mobile que foi desenvolvida.

\section{Aspectos de Implementação}

Desenvolver aplicações mobile em um ambiente nativo para cada plataforma existente no mercado requer uma demanda muito grande de tempo, esforço e custo, o que acaba impactando diretamente no produto final (Procópio e Pereira, 2015). Por outro lado, o desenvolvimento de aplicativos mobile multiplataforma permite uma única implementação de código, desenvolvida em HTML (HyperText Markup Language), CSS (Cascading Style Sheets) e JavaScript, podendo ser distribuída para as diferentes plataformas existentes no mercado com menor esforço de tempo e custo. o melhor do desenvolvimento mobile multiplataforma. Entretanto, a aplicação foi disponibilizada apenas para os dispositivos com o sistema operacional Android, devido ao grupo responsável pelo desenvolvimento do aplicativo já possuir licença para disponibilização da aplicação para esta plataforma.

A Figura 5 apresenta parte do código da aplicação através da ferramenta Brackets ${ }^{1}$. O Brackets é um editor de código open source, focado em design para web e muito utilizado por desenvolvedores que trabalham com as tecnologias mencionadas acima.

\section{Avaliação, Resultados e Discussão}

Com o objeto inicial de analisar a viabilidade da ferramenta proposta, uma prova de conceitos foi desenvolvida. Mesmo não apresentando o formalismo de um estudo experimental formal, a realização deste tipo de estudo contribuiu para uma avaliação preliminar da hipótese formulada, bem como para a verificação da viabilidade da solução. Esta experiência permitiu ainda obter evidências adicionais a respeito dos requisitos necessários para a construção de um serviço que pudesse atender ao problema levantado por esta pesquisa. Para isso, foi especificado um cenário real que envolvesse o uso da aplicação proposta.

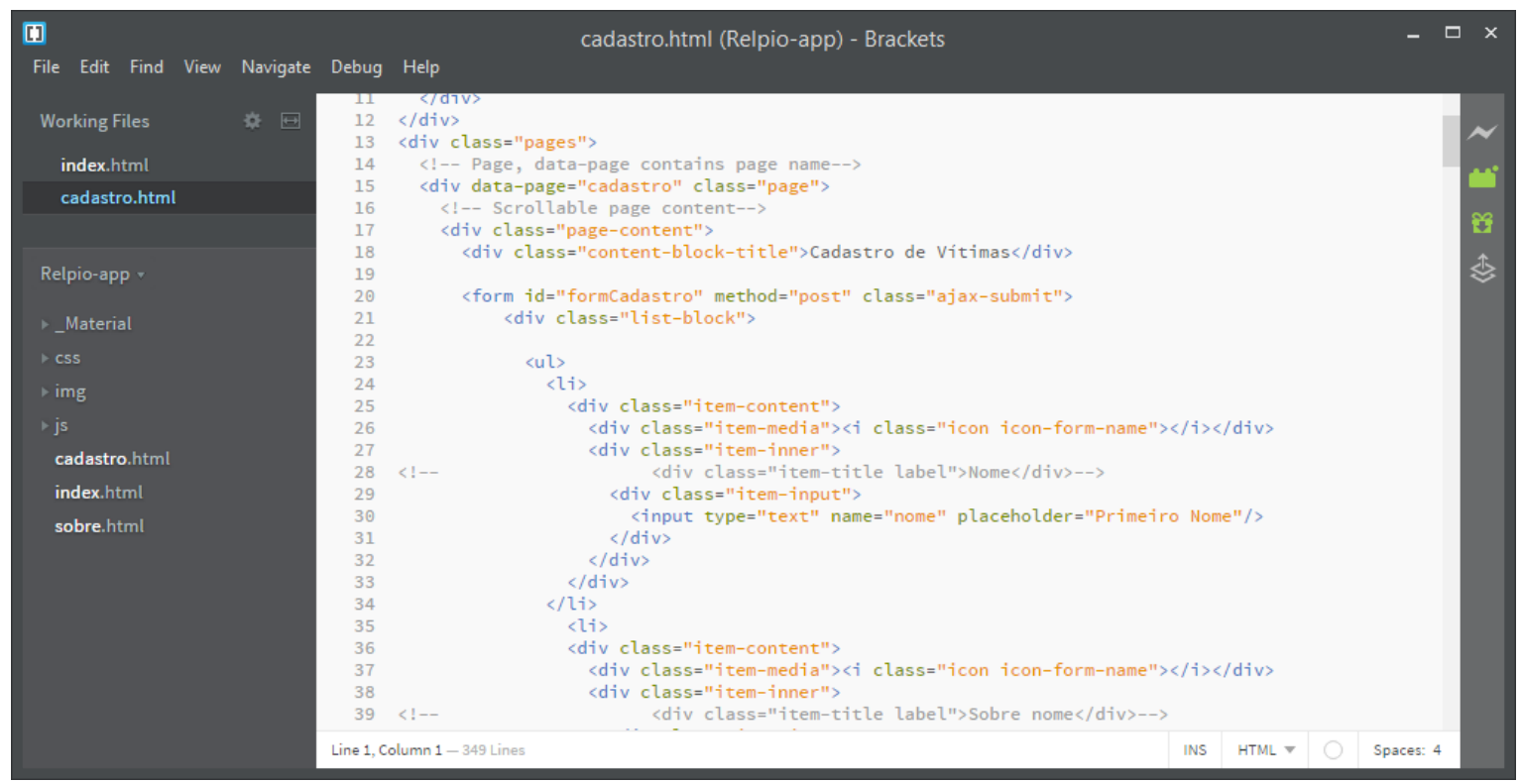

Figura 5. Parte do código da Tela de Cadastro de Vítimas

De acordo com Procópio e Pereira (2015), isso pode ser realizado através de um framework conhecido como Cordova, responsável por empacotar todo o código e gerar as versões dos aplicativos compatíveis com as principais plataformas do mercado, por exemplo: Android, IOS, Blackberry e Windows Phone.

Para isso, o Relpio foi desenvolvido utilizando as tecnologias destacadas acima, de forma a aproveitar
Neste cenário, uma vítima, pai de uma das famílias atingidas pela enchente, identificada pelo pseudônimo R, baixou o aplicativo Relpio em seu celular e realizou o seu cadastro na opção de cadastro de vítimas aproveitando a oportunidade para informar o ocorrido no seu bairro e a situação de seus familiares. Com estes dados disponibilizados no aplicativo, órgãos 1 Disponivel em: http://brackets.io/ 
responsáveis pela assistência social às famílias atingidas puderem entrar em contato com a vítima $R$, através do telefone informado no cadastro, validar as informações que foram passadas pela vítima, e ainda, direcionar a ajuda necessária à família.

Além disso, o órgão responsável por este atendimento, identificado aqui como órgão A.S.F pode manter atualizado o cadastro sobre os itens de doação de maior necessidade no momento, permitindo assim que outras pessoas, através do aplicativo pudessem conhecer locais de apoio e coleta de doações, e ainda, quais eram os itens que as famílias, vítimas da catástrofe, estavam necessitando com mais urgência.

Apesar da avaliação inicial apresentada neste estudo ter sido realizada através de uma prova de conceitos, passos concretos para a realização de estudos experimentais adicionais estão sendo dados. Entretanto, vale ressaltar que a realização da prova de conceitos apresentada colaborou de fato para a verificação da viabilidade da aplicação apresentada.

Alguns pontos negativos e que precisam ser melhorados na aplicação foram identificados ao longo da realização deste estudo. São eles: (i) a necessidade de conexão com a internet para a realização do cadastro de uma vítima, pode ser um obstáculo, visto que, nas duas cidades sobre a qual o estudo foi realizado, existem bairros em que não é possível uma conexão adequada com a internet, seja ela banda larga ou $3 \mathrm{G}$, por exemplo. Sendo assim algumas melhorias e outras estratégias precisam ser pensadas em relação a este ponto; (ii) a usabilidade da aplicação. Para este ponto um estudo de caso foi realizado com o objetivo de levantar os principais aspectos que precisariam ser melhorados. Este tópico é apresentado na subseção seguinte. (iii) adicionar novas funcionalidades à ferramenta. Este ponto também está sendo estudado, e alguns trabalhos futuros são apresentados na última seção deste artigo.

Buscando anemizar o problema da usabilidade da aplicação, um estudo de caso inicial foi realizado e é apresentado na seção 3.1 .

\section{Estudo de Caso}

O objetivo da realização deste estudo de caso inicial foi analisar a usabilidade da aplicação em relação à facilidade de uso das funcionalidades oferecidas pelo aplicativo. Tudo isso, sob o ponto de vista de usuários comuns, buscando conhecer mais detalhes e encontrar pontos de melhorias que pudessem ser corrigidos ou melhorados na ferramenta.

Para a realização deste estudo de caso, antes da publicação e distribuição da aplicação na loja oficial de aplicativos da Google, uma cópia da versão 1.0.0 da aplicação foi disponibilizada para um avaliador, identificado aqui como avaliador $\mathrm{F}$.
O avaliador $F$ é um profissional de desenvolvimento de software de uma software house localizada na cidade de Três Rios e com aproximadamente dois anos de experiência na área. De posse da aplicação, o usuário $\mathrm{F}$ pode usar a aplicação assumindo os dois papeis inicias pensados para esta aplicação: (i) usuário vítima da catástrofe e que desejasse realizar o seu cadastro no aplicativo; e (ii) um usuário que procura pontos de apoio para realizar a doação para famílias atingidas pelo evento. Isso, sob o ponto de vista de um usuário inicial que teve acesso a primeira vez ao aplicativo. A duração da avaliação foi de aproximadamente 20 minutos. A cada passo que o avaliador F usava a aplicação, o seu ponto de vista era manifestado através do registro de um novo tópico que ele realizava. Um observador (primeiro autor deste artigo) observava a interação e o uso por parte do avaliador F com o aplicativo Relpio. As observações feitas pelo avaliador F podem ser acessadas através do endereço: https://goo.gl/RtDtMD.

Mesmo se tratando de um estudo de caso inicial, a sua realização colaborou para que o grupo pudesse ter um olhar mais voltado para o usuário final da aplicação. Apesar de ser um estudo ainda muito inicial e sem todo o rigor necessário de um estudo experimental formal, grande parte as observações feitas pelo avaliador $\mathrm{F}$ já foram corrigidas ou melhoradas na versão 1.0.1 do Relpio, disponível através da Play Store.

Novos estudos estão sendo elaborados para serem aplicados, bem como novas funcionalidades tem sido estudada para serem desenvolvidas e aplicadas na ferramenta.

\section{Considerações Finais e Trabalhos Futuros}

Este trabalho apresentou um aplicativo mobile desenvolvido para a plataforma Android com o objetivo de auxiliar no cadastro das vítimas de catástrofes naturais, de forma a apoiar órgãos responsáveis na oferta de atendimento a estas famílias logo nas primeiras horas do evento. Trata-se de um aplicativo ainda em fase inicial, podendo ser considerado um protótipo, visto o número de possíveis trabalhos futuros que ainda podem ser desenvolvidos pelo grupo, tais como: (i) adicionar relatórios analíticos para mostrar aos órgãos que utilizam o aplicativo os lugares onde a população foi mais afetada, baseado nas vítimas cadastradas; (ii) possibilitar a gravação dos cadastros das vítimas sem utilizar a internet (off-line), e só enviar esse cadastro para o web service quando conseguir conectar à internet. Desta forma é possível que o aplicativo funcione em locais sem acesso à internet, possibilitando que agentes da defesa civil possam ir até às vítimas em locais de difícil acesso; (iii) criar um sistema central que possibilite 
realizar mais operações como cadastro de usuários, agentes, oferecendo mais controle e personalização para necessidade; entre outros.

Como trabalhos futuros ainda, acredita-se que explorar como comunidades locais de desenvolvedores podem apoiar e ajudar o desenvolvimento de sua cidade pode ser um ponto importante, visto que iniciativas como esta pode levar uma cidade ou mesmo uma comunidade a se desenvolver como uma comunidade/ cidade inteligente.

Algumas das contribuições observadas e que podem ser alcançadas através do uso da aplicação são: (i) contribuir para melhorar a resiliência dos cidadãos diante de situações de catástrofe; (ii) possibilitar aos órgãos responsáveis um melhor planejamento de ação baseado nos dados coletados do aplicativo; (iii) permitir que os agentes da defesa civil possam realizar o cadastro das vítimas no próprio local, agilizando o levantamento dos dados, entre outros.

Por fim, a aplicação prática do Relpio em contextos similares ao apresentado neste trabalho pode permitir, mesmo que com um apoio menor, o atendimento e a prestação de ajuda às famílias atingidas por catástrofes naturais possam ser ofertados no logo primeiro momento de uma situação de emergência, podendo ainda minimizar o acontecimento de situações mais graves.

\section{Referências}

Allen, S., Graupera, V., \& Lundrigan, L. (2010). Pro Smartphone CrossPlatform Development: iPhone, Blackberry, Windows Mobile and Android Development and Distribution. (1st ed). New York: Apress.

Cordeiro, K.F.; Marino, T., Campos, M.L.M.; Borges, M.R.S. (2011) Use of Linked Data in the Design of Information Infrastructure for Collaborative Emergency Management System. International Conference on Computer Supported Cooperative Work in Design (CSCWD). Swiss.

De Faria Cordeiro, Kelli; Campos, Maria Luiza Machado; Da Silva Borges, Marcos Roberto. (2014). Adaptive Integration of Information Supporting Decision Making: A Case on Humanitarian Logistic. Proceedings of ISCRAM 2014.

Education, U. S. D. O. (2010). Action Guide for Emergency Management At Institutions of Higher Education. Acesso em: 27/01/2016, from http://rems. ed.gov/docs/REMS_ActionGuide.pdf

FEMA, Developing and Maintaining Emergency Operations Plans: Comprehensive Preparedness Guide (CPG) 101. 2 v. 2010.

Lage, B. B.; Borges, M. R. S.; Canós, J. H.; Vivacqua, A. S. (2011). Facilitating collaborative scenario creation to support emergency plan generation. In: Fifteenth International Conference on Computer Supported Cooperative Work in Design (CSCWD), Junho 2011, Lausanne, Switzerland. Proceedings. Estados Unidos: IEEE, 2011, p. 657-664.

Procopio, L. L.; Pereira, A. F. (2015). Buscando agilidade no desenvolvimento de aplicações Mobile através do desenvolvimento multiplataforma. In: XIV Encontro de Iniciação Científica - USS, 2015, Vassouras / RJ. Anais do XIV Encontro de Iniciação Científica. Vassouras: Editora USS, 2015. p. 173173.

Souza, F., \& Kushchu, I. (2005). Mobile Disaster Management System Applications-Current Overview And Future Potential - Mlife conference \& exhibitions.

Wattegama, C (2007). ICT for disaster management, United Nations
Development Programme-Asia-Pacific Programme (UNDP-APDIP).
Development Information 